\title{
Numerical optimization of a plasma wakefield acceleration experiment
}

\author{
K. V. Lotov and V.S. Tikhanovich \\ Budker Institute of Nuclear Physics, 630090, Novosibirsk, Russia, \\ and Novosibirsk State University, 630090, Novosibirsk, Russia \\ (Received 2 March 2007; published 14 May 2007)
}

\begin{abstract}
One possible way to demonstrate both the efficiency and beam quality in a plasma wakefield accelerator is to prepare matched drive and accelerated beams by removing a central slice from a single high-quality electron bunch (parent beam). For parameters of the parent beam given, the question arises how to maximize the number and energy of accelerated particles and minimize their energy spread and emittance. This question is addressed by numerical simulations. The optimum shape of the beams, required plasma length, achievable energy gain, and energy spread are found as functions of the plasma density and parent beam characteristics. The required control accuracy of adjustable beam and plasma parameters is determined.
\end{abstract}

DOI: 10.1103/PhysRevSTAB.10.051301

PACS numbers: 52.40.Mj, 41.75.Lx, 07.05.Tp

\section{INTRODUCTION}

Since the mid-1980s [1], the electron beam-driven plasma wakefield acceleration (PWFA) is actively studied as a possible way to future high-energy linear colliders [25]. Already demonstrated are good agreement between theoretical predictions and experimental observations [3,6-11], high acceleration rate, and high-energy gain [12-14]. High efficiency of beam-to-beam energy transfer and high quality of the accelerated beam (witness) are not experimentally proven yet; this should be the next major step toward a competitive plasma-based accelerator.

One possible way to achieve both the efficiency and beam quality is to prepare matched drive and accelerated beams by removing a central slice from a single highquality electron bunch (parent beam, Fig. 1). Given the parameters of the parent beam, the question arises how to maximize the number and energy of accelerated particles and minimize their energy spread and emittance. Other questions are what characteristics of the witness can be achieved with available parent beams and to what accuracy the adjustable parameters of the system must be held.

These questions are addressed in the paper. In Sec. II we formulate the optimization problem to be solved. In Sec. III we discuss the optimum values for adjustable parameters of the beam and plasma. In Sec. IV we analyze the accuracies required. In Sec. V we summarize main findings.

It is important to emphasize the degree of generality of the obtained results. As a reference point for optimization, we take the design parameters of PWFA experiment on the VEPP-5 injection complex $[15,16]$. The numerical values obtained are therefore applicable to this experimental project only. The dimensionless relations are more general and valid for any experiment where the parent beam has a Gaussian-like shape. The scalings (Sec. III) and order-ofmagnitude estimates for accuracies (Sec. IV) are fully general and applicable wherever the driver and accelerated beam are cut from a single beam.

\section{OPTIMIZATION PROBLEM}

There are two groups of input parameters in our problem. Parameters from the first group (Table I) are stringently determined by the accelerator that produces the parent beam and by the system that compresses the parent beam and splits it into two pieces. These parameters cannot be easily varied and are not a subject of optimization; we take them close to the design parameters of Novosibirsk PWFA experiment $[15,16]$. Parameters from the second group (Table II) can be adjusted relatively easily; optimum values of them are to be found.

Assume the parent beam at the entrance to the plasma is axisymmetric with the density distribution

$$
\begin{aligned}
n_{p b} & =\frac{N_{p}}{2 \sigma_{r}^{2} \sigma_{z}(2 \pi)^{3 / 2}} e^{-r^{2} / 2 \sigma_{r}^{2}}\left[1+\cos \left(\sqrt{\frac{\pi}{2}} \frac{z}{\sigma_{z}}\right)\right], \\
|z| & <\sigma_{z} \sqrt{2 \pi},
\end{aligned}
$$

if no slices are cut out. The cosine distribution (1) over the longitudinal coordinate $z$ is more convenient than the Gaussian one because it smoothly vanishes outside the interval of a finite length. With the cutting on, the initial beam density is

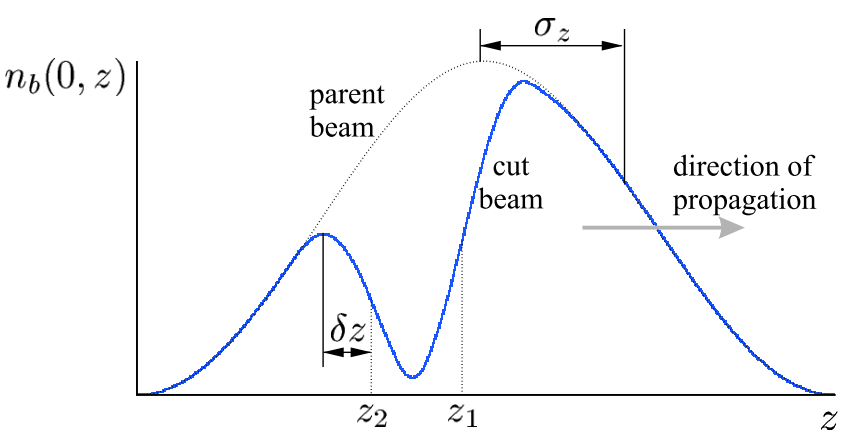

FIG. 1. (Color) Beam shape at the entrance to the plasma. 
TABLE I. Fixed parameters of the beam.

\begin{tabular}{lc}
\hline \hline \multicolumn{1}{c}{ Parameter and notation } & Value \\
\hline Initial energy, $W_{0}$ & $510 \mathrm{MeV}$ \\
Number of particles in the parent beam, $N_{p}$ & $2 \times 10^{10}$ \\
Normalized rms emittance, $\epsilon_{0}$ & $20 \mathrm{~mm} \mathrm{mrad}$ \\
Initial energy spread, $\delta W_{0}$ & $0 \%(\mathrm{low})$ \\
Minimum possible length, $\sigma_{z, \text { min }}$ & $0.1 \mathrm{~mm}$ \\
Minimum possible radius, $\sigma_{r, \text { min }}$ & $20 \mu \mathrm{m}$ \\
Cut-out sharpness, $\delta z$ & $0.1 \mathrm{~mm}$ \\
\hline \hline
\end{tabular}

TABLE II. Adjustable parameters of the system.

\begin{tabular}{lc}
\hline \hline \multicolumn{1}{c}{ Parameter and notation } & Limitation \\
\hline Compressed beam length, $\sigma_{z}$, & $\sigma_{z} \geq \sigma_{z, \text { min }}$ \\
Projections of chopper edges onto & $z_{1}>z_{2}$ \\
$\quad$ the compressed beam, $z_{1}$ and $z_{2}$, & \\
Beam radius, $\sigma_{r}$, & $\sigma_{r} \geq \sigma_{r, \text { min }}$ \\
Plasma density, $n_{0}$ & \\
Plasma length, $L_{p}$ & \\
\hline \hline
\end{tabular}

$$
n_{b}(r, z)=n_{p b}\left[g\left(z, z_{2}\right)+1-g\left(z, z_{1}\right)\right],
$$

where the function $g\left(z, z_{i}\right)$ describes a smooth cut-out edge:

$$
g\left(z, z_{i}\right)= \begin{cases}1, & z<z_{i}-\delta z \\ 0.5\left(1-\sin \frac{\pi\left(z-z_{i}\right)}{2 \delta z}\right), & \left|z-z_{i}\right|<\delta z \\ 0, & z>z_{i}+\delta z\end{cases}
$$

The geometry of the cut beam is illustrated by Fig. 1 .

The initial angular spread of the beam is chosen the same for both transverse coordinates to stay within the axisymmetric approximation, so the initial 6-dimensional distribution function of beam particles is

$$
f(\vec{r}, \vec{p}) \propto n_{b}(r, z) \exp \left(-\frac{\left(p_{r}^{2}+p_{\varphi}^{2}\right) \sigma_{r}^{2}}{2 \epsilon_{0}^{2} m^{2} c^{2}}\right) \delta\left(p_{z}-\gamma_{0} m c\right),
$$

where $\gamma_{0}=W_{0} /\left(m c^{2}\right)=1000$, and $\delta$ is the delta function.

Dynamics of thus defined beam in the uniform unmagnetized plasma of the length $L_{p}$ and density $n_{0}$ is followed numerically with LCODE code $[17,18]$. Typical parameters of the run are listed in Table III; they are chosen to maximize the computation speed at a reasonable precision. As can be seen, the time step and sizes depend on the plasma frequency $\omega_{p}=\sqrt{4 \pi n_{0} e^{2} / m}$.

The output of the code is a 6-space distribution of beam macroparticles at the exit from the plasma. From these data, we find four goal parameters: the number of accelerated particles $N_{w}$, their average energy gain $\Delta W$, rms normalized emittance $\epsilon_{w}$, and energy spread $\delta W$. To exclude a possible halo, only particles that meet several
TABLE III. Parameters of a typical LCODE run.

\begin{tabular}{lc}
\hline \hline \multicolumn{1}{c}{ Parameter } & Value \\
\hline Plasma model & Kinetic, quasistatic \\
Number of plasma macroparticles & 800 \\
Initial plasma temperature & $5 \mathrm{eV}$ \\
Radius of simulation window & $4 c / \omega_{p}$ \\
Length of simulation window & $10 c / \omega_{p}$ \\
Grid size & $0.025 c / \omega_{p} \times 0.025 c / \omega_{p}$ \\
Plasma length & $6000 c / \omega_{p}$ \\
Number of beam macroparticles & $10^{4}$ \\
Time step for the beam & $2 \omega_{p}^{-1}$ \\
\hline \hline
\end{tabular}

selection criteria are taken into account. These criteria are: the particle must originate from the second beam [from $z<\left(z_{1}+z_{2}\right) / 2$ ], have a small final radius $(r<$ $\left.\sigma_{r}\right)$, small angle of departure $\left[\left|p_{r}\right| / p_{z}<\epsilon_{0} /\left(\gamma_{0} \sigma_{r}\right)\right]$, and the energy in the $30 \%$ vicinity of the spike of final energy distribution $\left[\left|\left(p_{z} c-W_{0}\right) / \Delta W-1\right| \lesssim 0.3\right]$.

For realization of the optimization algorithm, it is necessary to combine the goal parameters into a single-valued criterion function $F$. The choice of the criterion function determines the convergence speed and, in principle, can strongly affect the result of optimization procedure. Fortunately, in the problem considered, there exists an acceleration regime for which all goal parameters are simultaneously good and there is no trade-off between, for example, energy gain and number of particles. Thus, the result weakly depends on the particular criterion function if the latter is reasonably chosen. Among several criterion functions tested, the function

$$
\begin{aligned}
F= & \ln \left(N_{w} / N_{p}\right)+\ln \left(\Delta W / W_{0}\right)+\frac{1}{1+\left(\frac{\epsilon_{w}}{2 \epsilon_{0}}\right)^{2}} \\
& +\frac{1}{1+\left(\frac{\delta W}{0.1 \Delta W}\right)^{2}}
\end{aligned}
$$

provides the best convergence. Here, the logarithms prohibit too small values of maximized quantities and moderately stimulate their increase in the vicinity of expected values (about $N_{p} / 5$ for the population and $W_{0}$ for the energy gain). The rational functions flatten the contributions of minimized quantities if they are much smaller than some "acceptable" values ( $2 \epsilon_{0}$ for the final emittance and $0.1 \Delta W$ for the energy spread) to avoid overminimization at the expense of beam population. If the minimized quantities are too large, their contributions are also flattened to avoid a delay of the search algorithm in the region of obviously unacceptable parameters. In the paper, all the results presented are obtained with this criterion function.

The above procedure defines the function $F\left(\sigma_{r}, \sigma_{z}\right.$, $\left.z_{1}, z_{2}, n_{0}, L_{p}\right)$ that has to be maximized in the 6-space of adjustable parameters. The function is noisy; a small variation of any argument can result in a large change of the value. This is because beam particles make about $10^{2}$ 
betatron oscillations in a typical plasma length, and a very small variation of run parameters is sufficient to change the oscillation phase at the exit. Because of the noise, the search of maxima is best done with a simple step-by-step algorithm. The search starts from some random point and moves alternately in each coordinate until a local maximum is found with a given precision. Typically, one search takes about 200 code runs and 50 hours at Pentium-IV.

Because of the noise, the search started from different points results in different local maxima. The local maxima found are not uniformly distributed in the parameter space; they concentrate in regions of high $F$ thus showing not only the location of optimum regimes in the parameter space but also the widths of these regimes. Local maxima with low values of $F$ can be easily culled.

\section{OPTIMUM REGIME}

The main result of the above optimization is that the optimum parameters form a curve in the 6-space of adjustable parameters. The curve has one free parameter; let it be the plasma density $n_{0}$. Other adjustable parameters are thus determined by $n_{0}$ :

$$
\begin{gathered}
\sigma_{z} \approx 2.15 c / \omega_{p}, \quad \sigma_{r}=\sigma_{r, \min }, \\
z_{1} \approx 0, \quad z_{2} \approx-\frac{\pi}{2} c / \omega_{p}, \quad L_{p} \approx A \gamma_{0} c / \omega_{p},
\end{gathered}
$$

where the factor $A$ depends on the parent beam population. In our case $A \approx 5$. Figure 2 illustrates these statements. From Fig. 2(a) we can see that, though $F \neq$ const on the optimum curve, this inconstancy is small compared to the decrease of $F$ as its arguments deviate from the curve.

It is instructive to look at the evolution of main witness parameters in the plasma (Fig. 3). In this section, we use the input parameter set marked by crosses in Fig. 2 and apply no halo filtering criteria. We see that the beam evolution occurs in three stages. After escape of the beam tail [Fig. 3(b)], the rest of the witness stably propagates in the plasma until the driver gets considerably depleted [Fig. 3(d)]. Then the driver widens, the wakefield structure changes, and the witness gets defocused and lost [Fig. 3(b)]. The end of the second stage corresponds to the optimum plasma length. The increase of emittance during the second stage is a numerical effect that disappears at shorter time steps, so it is a price for fast computing.

The resulting energy spectrum depends on the plasma density (Fig. 4). The witness energy gain is limited to about the initial driver energy. At high plasma densities, the energy gain is high, but the energy spread is large because of the limited beam shape control [Fig. 4(b)]. Namely, as the density increases, the width of the cut-out interval gets smaller than the cut-out sharpness $\delta z$, and the witness energy spread increases [Fig. 5(a)]. In units of $e E_{0} \equiv$ $m c \omega_{p}$, the average accelerating rate is constant in a wide interval of plasma densities [Fig. 5(b)] and equals $A^{-1}$. Together with the expression (5) for $L_{p}$, this suggests that the acceleration distance here is determined by the driver depletion. At low plasma densities, the acceleration length is limited by emittance-driven driver erosion, much of the energy remains with the driver [Fig. 4(b)], and the witness
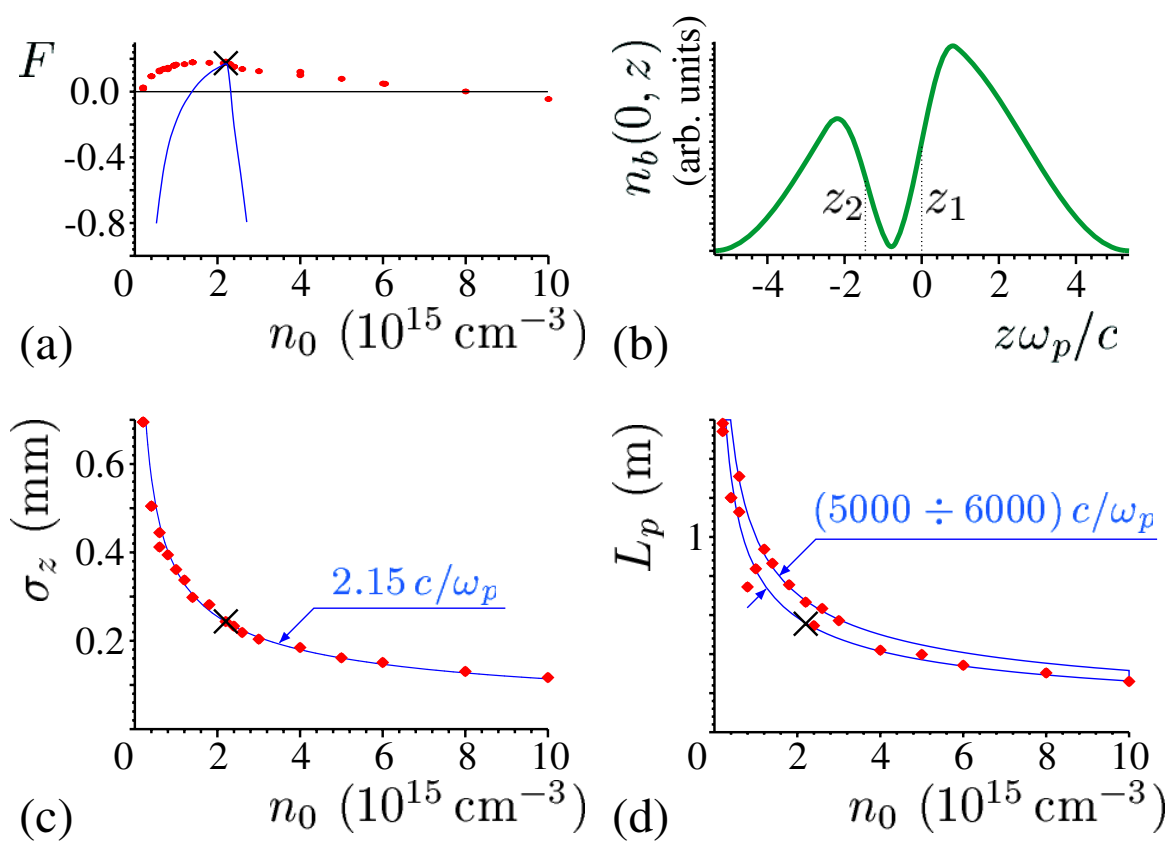

FIG. 2. (Color) (a) Values of the criterion function on the optimum curve (dots) and on the line $\left(\sigma_{r}, \sigma_{z}, z_{1}, z_{2}, L_{p}\right)=$ const (line); (b) optimum beam shape; (c) optimum beam length; and (d) optimum plasma length. Dots show the local maxima obtained numerically. Crosses mark the regime under the detailed study. 

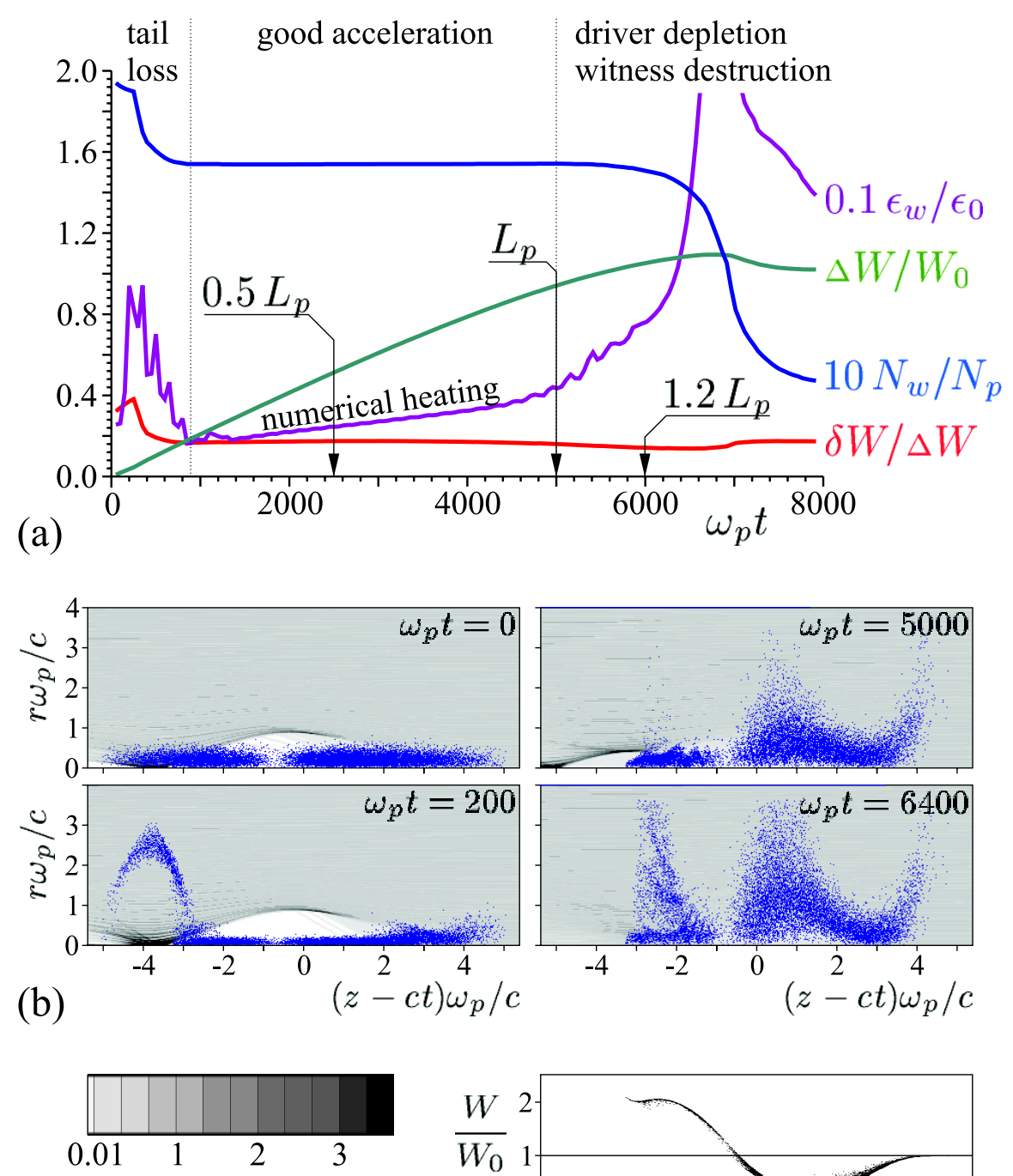

(c)

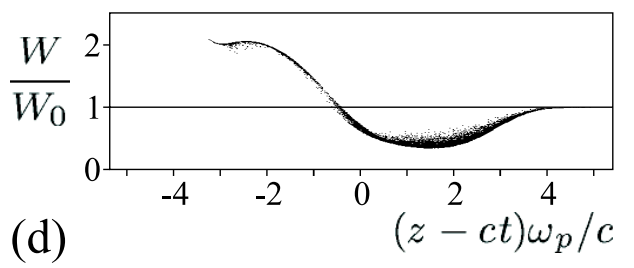

FIG. 3. (Color) (a) Evolution of witness emittance $\epsilon_{w}$, energy gain $\Delta W$, population $N_{w}$, and energy spread $\delta W$ as the witness propagates in the plasma; (b) portraits of the beam (dots) and plasma electron density (shading) at different times; (c) the palette for electron density maps; (d) the dependence of the beam energy $W$ on the longitudinal coordinate at the end of good acceleration (at $\left.\omega_{p} t=5000\right)$.

energy gain is small. Thus, the optimum plasma density is the minimum one at which the acceleration length is determined by driver depletion $\left(n_{0} \approx 2.2 \times 10^{15} \mathrm{~cm}^{-3}\right.$ in our case).

The decrease of the average acceleration rate at low plasma densities [Fig. 5(b)] follows from properties of the blowout regime. As the plasma density decreases, the beam lengthens in accordance with (4) and the beam peak current $I_{b, \max }$ decreases as $\sqrt{n_{0}}$. In the problem considered, typical beam currents are not very high $\left(I_{b, \max } \sim 0.5 \div\right.$ $2 \mathrm{kA}$ ), so the dimensionless bubble radius is determined by the beam current $I_{b}$ as [19]

$$
\frac{r_{\mathrm{cav}} \omega_{p}}{c} \approx B \sqrt{\frac{I_{b}}{17 \mathrm{kA}}},
$$

where the factor $B \approx 2.8$ for $\sigma_{z} \omega_{p} / c \approx 2.15$. The same relation holds for the maximum bubble radius:

$$
r_{\max } \approx B \frac{c}{\omega_{p}} \sqrt{\frac{I_{b, \max }}{17 \mathrm{kA}}} .
$$

Expressing $I_{b, \max }$ in terms of beam population $N_{b}$ and substituting the optimum value (4) for $\sigma_{z}$ yields 


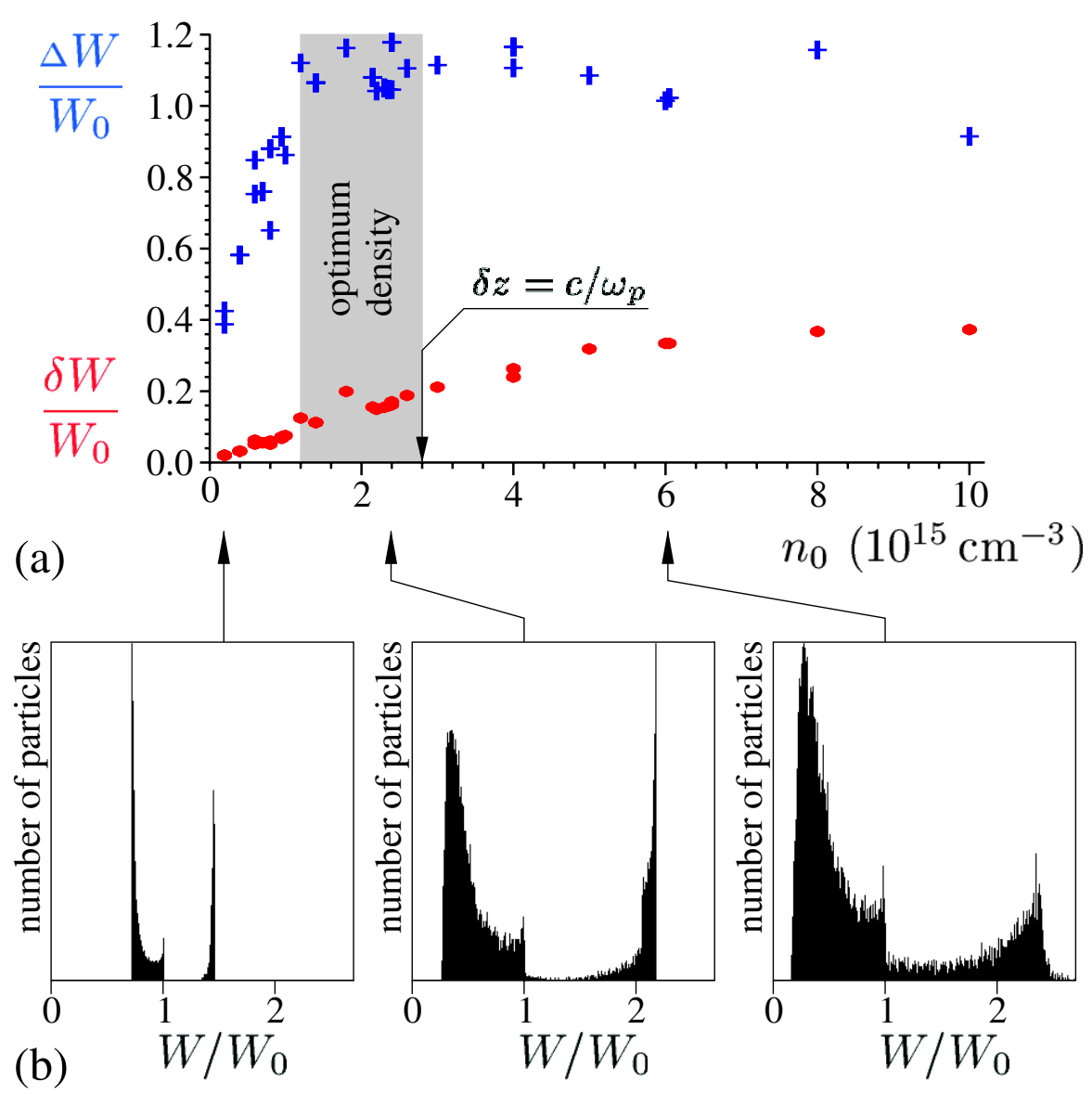

FIG. 4. (Color) (a) Dependence of the witness energy gain $\Delta W$ (crosses) and energy spread $\delta W$ (ovals) on the plasma density; (b) typical energy spectra at various densities.

$$
r_{\max } \approx B \frac{c}{\omega_{p}} \sqrt{\frac{e^{2} N_{p}}{m c^{2} \sigma_{z} \sqrt{2 \pi}}} \approx 0.64 \sqrt{N_{p} \sqrt{\frac{r_{e}}{n_{0}}}}
$$

where $r_{e}=e^{2} /\left(m c^{2}\right)$ is the classical electron radius. The dimensionless bubble radius is

$$
\frac{r_{\max } \omega_{p}}{c} \approx 2.3 \sqrt{N_{p} \sqrt{n_{0} r_{e}^{3}}}
$$
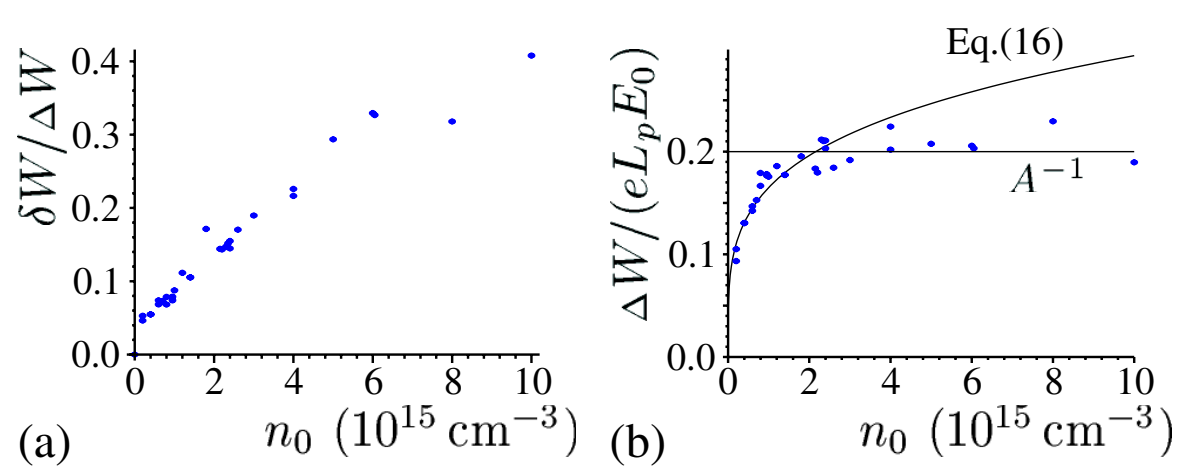

FIG. 5. (Color) Dependence of the relative witness energy spread (a) and dimensionless average accelerating rate (b) on the plasma density.

The scaling (9) is very accurate at early stages of beamplasma interaction [Fig. 6(a)].

At the beam energies considered, we can safely put

$$
\frac{\partial}{\partial z}=-\frac{1}{c} \frac{\partial}{\partial t}
$$

In this approximation, we can obtain from Maxwell equations the expression for the on-axis electric field (see, e.g., [19]): 

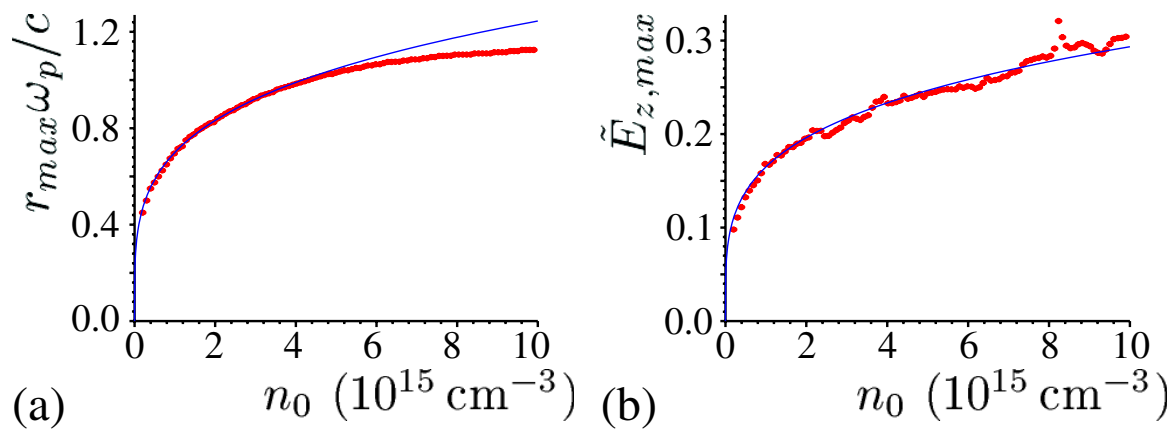

FIG. 6. (Color) Plasma density dependence of the maximum bubble radius (a) and maximum decelerating field (b) at the early stage of beam-plasma interaction. Dots show numerically obtained values, curves are obtained from formulas (11) and (17).

$$
E_{z}(0, z-c t)=\frac{4 \pi e}{c} \int_{0}^{\infty} n v_{r} d r
$$

where $n=n(r, z-c t)$ and $v_{r}=v_{r}(r, z-c t)$ are the density and radial velocity of plasma electrons. If the bubble is narrow compared to the plasma skin depth $c / \omega_{p}$ (as it is in our case), then there is no electron density peaking at the bubble boundary and the radial scale of plasma perturbations is $c / \omega_{p}$ :

$$
n \approx n_{0}, \quad \int \ldots d r \sim c / \omega_{p}
$$

It is natural to assume that the maximum radial velocity of plasma electrons is proportional to their radial shift and inversely proportional to the time scale of motion:

$$
v_{r} \propto \frac{c r_{\max }}{\sigma_{z}} \propto n_{0}^{1 / 4},
$$

whence follows the scaling for the maximum decelerating electric field inside the bubble:

$$
E_{z, \max } \propto n_{0}^{3 / 4}
$$

The proportionality coefficient can be easily obtained from simulations. For the dimensionless field,

$$
\tilde{E}_{z, \max } \equiv \frac{e E_{z, \max }}{m c \omega_{p}} \approx 0.165\left[n_{0}, 10^{15} \mathrm{~cm}^{-3}\right]^{1 / 4}
$$

The maximum field observed in simulations follows the scaling (15) very good [Fig. 6(b)] as does the average field at low plasma densities [Fig. 5(b)]. At high plasma densities, the decelerating field considerably decreases as the driver propagates in the plasma, so the average field is lower than the maximum field achieved near the entrance to the plasma, and we observe the saturation of the energy gain at high plasma densities.

The utilization efficiency of the driver energy is shown in Fig. 7. Since the deceleration field is not uniform within the beam, about $50 \%$ of the energy is left with the driver. An extra $20 \%-30 \%$ is left in the plasma, so the overall driver-to-witness efficiency is at the $30 \%$ level in the wide interval of plasma densities.

Note that the beam-plasma interaction in the optimum regime has most of the features of the efficient acceleration mode described in Ref. [20]. Namely, both drive and witness beams propagate in the blowout regime [21] in the electron-free bubble, the beams are shaped to flatten decelerating or accelerating field inside of them, and the length of the driver is about one wakefield half-wavelength. The only missed feature is the beam current, the relatively low value of which limits the efficiency at the $30 \%$ level.

\section{ACCURACIES REQUIRED}

Once the optimum set of adjustable parameters is found, the question arises of how precisely these values must be

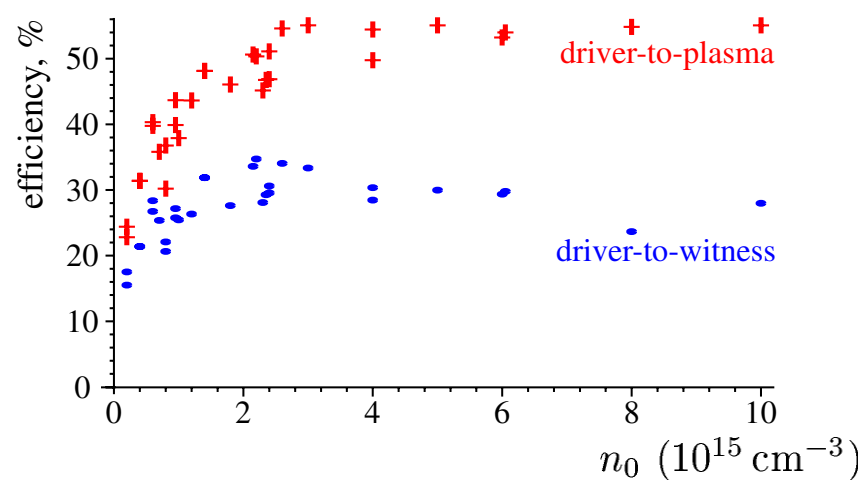

FIG. 7. (Color) Efficiency of driver-to-plasma (crosses) and driver-to-witness (ovals) energy transfer. 
TABLE IV. Acceptable deviation of adjustable parameters from the optimum values.

\begin{tabular}{|c|c|c|c|c|}
\hline Limiting factor & Downward tolerance & Parameter & Upward tolerance & Limiting factor \\
\hline $\begin{array}{c}\text { Incomplete } \\
\text { energy exchange }\end{array}$ & $-50 \%$ & Plasma length $L_{p}$ & $+20 \%$ & $\begin{array}{c}\text { Driver } \\
\text { depletion }\end{array}$ \\
\hline $\begin{array}{l}\text { Mismatched } \\
\text { witness }\end{array}$ & $-20 \%$ & Beam length $\sigma_{z}$ & $+25 \%$ & $\begin{array}{l}\text { Witness beyond } \\
\text { the bubble }\end{array}$ \\
\hline $\begin{array}{l}\text { Mismatched } \\
\text { witness }\end{array}$ & $-25 \%$ & Plasma density $n_{0}$ & $+20 \%$ & $\begin{array}{c}\text { Driver depletion } \\
\text { (increase of effective } \\
\text { plasma length) }\end{array}$ \\
\hline $\begin{array}{l}\text { Witness beyond } \\
\text { the bubble }\end{array}$ & 0.45 back & $\begin{array}{c}\text { Relative shift of } \\
\text { the cut-out interval } \\
0.5\left|z_{1}+z_{2}\right| /\left(z_{1}-z_{2}\right)\end{array}$ & 0.45 forth & $\begin{array}{c}\text { Small driver, } \\
\text { mismatched witness }\end{array}$ \\
\hline $\begin{array}{l}\text { Mismatched } \\
\text { witness }\end{array}$ & $-30 \%$ & Cut-out interval width $\left(z_{1}-z_{2}\right)$ & $+30 \%$ & $\begin{array}{l}\text { Witness beyond } \\
\text { the bubble }\end{array}$ \\
\hline
\end{tabular}

controlled. To answer this question, we follow the variation of main witness parameters as one of adjustable parameters of the system deviate from the optimum value (marked by crosses in Fig. 2). The results are listed in Table IV and illustrated by Figs. 3(a) and 8. The tolerances listed correspond to a twofold increase/decrease of any minimized/ maximized witness parameter (marked by continuous lines in Fig. 8).

There are essentially three major scenarios of witness degradation. The first two are related to the mismatch between the beam and the wakefield. Variation of any input parameter, except the plasma length, can cause this mis-

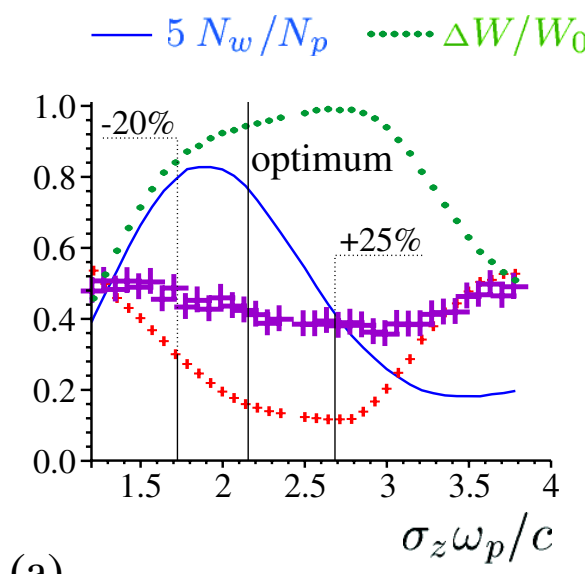

(a)

世冊 $0.1 \epsilon_{w} / \epsilon_{0}{ }^{++++++} \delta W / \Delta W$
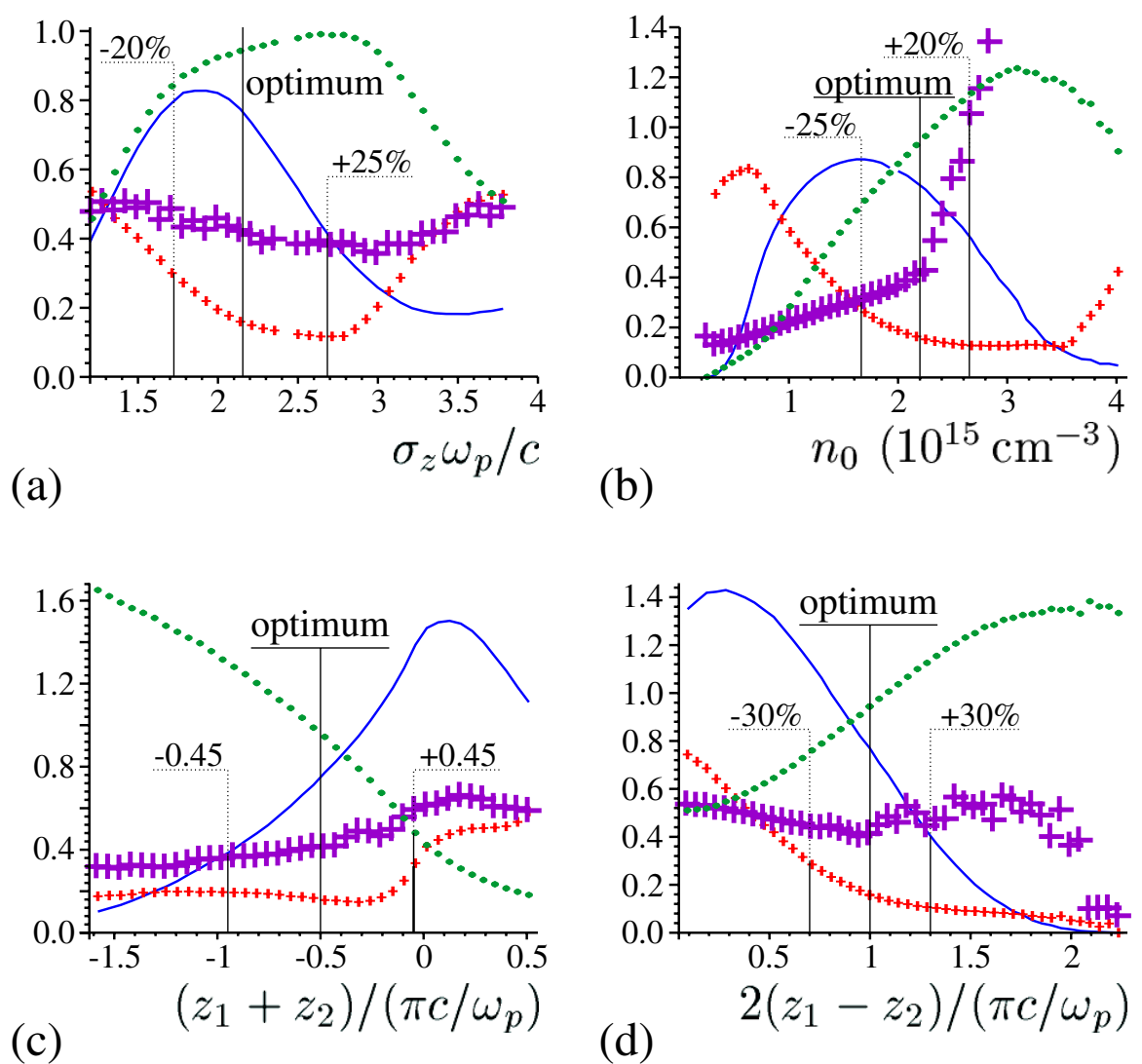

(d)

FIG. 8. (Color) Variation of the witness population $N_{w}$, energy gain $\Delta W$, emittance $\epsilon_{w}$, and energy spread $\delta W$ as (a) the parent beam length, (b) plasma density, (c) cut-out location, and (d) cut-out width deviate from the optimum values. 
match. Depending on the sign of the effect, the witness either gains a large energy spread ("mismatched witness") or shifts to the defocusing region and loses good emittance or population ("witness beyond the bubble"). The third scenario ("driver depletion") takes place if the depleted driver gets destroyed before the beam exits the plasma. This happens not only in too long, but also in overdense plasmas, since it is the dimensionless plasma length that is to match the optimum value.

We see that most of adjustable parameters of the system need to be controlled with $20 \%-30 \%$ accuracy, and the most sensitive parameter is the plasma density. Note also that an overlength plasma in much worse than an underlength one.

\section{SUMMARY}

Present-day computers make possible a multiparametric optimization of plasma wakefield accelerators, which includes a two-dimensional end-to-end simulation of beam dynamics in the plasma section as the elementary step. The optimum seeking, however, is complicated by a noise of the maximized function.

If both the driver and witness beams are cut from a single parent beam, then only one parameter of the system can be chosen relatively freely, for example, the plasma length or density. Once this parameter is specified, other ones are uniquely determined. For the Gaussian-like shape of the parent beam, relations between the adjustable parameters of the system are given by formulas (4) and (5). The optimum plasma density is the minimum one at which the length of beam-plasma interaction is determined by the driver depletion rather than by emittance-driven erosion.

The witness energy gain achievable with the Gaussian parent beam is limited by about the parent beam energy. The energy spread is greater than 5\% and increases with the plasma density.

The region of optimum values in the space of adjustable parameters is rather broad. The control accuracy required is at the $20 \%-40 \%$ level.

\section{ACKNOWLEDGMENTS}

The authors are grateful to A.M.Kudryavtsev, N.N. Lebedev, A. V.Petrenko, V.B. Reva, T. V. Rybitskaya, A. A. Starostenko, and S. Yu. Taskaev for granting their computing resources to perform this study. This work was supported by Russian Foundation for Basic Research (Grants No. 03-02-16160, No. 05-02-16775, No. 06-02-16757), SB RAS grant for young scientists, and President Grants No. NSh-2749.2006.2 and No. MD4704.2007.2.

[1] P. Chen, J. M. Dawson, R. W. Huff, and T. Katsouleas, Phys. Rev. Lett. 54, 693 (1985).

[2] E. Esarey, P. Sprangle, J. Krall, and A. Ting, IEEE Trans. Plasma Sci. 24, 252 (1996).

[3] C. Joshi et al., Phys. Plasmas 9, 1845 (2002).

[4] T. Katsouleas, Plasma Phys. Controlled Fusion 46, B575 (2004).

[5] T. Katsouleas, Phys. Plasmas 13, 055503 (2006).

[6] J. B. Rosenzweig et al., Phys. Rev. Lett. 61, 98 (1988).

[7] J. B. Rosenzweig et al., Phys. Rev. A 39, 1586 (1989).

[8] C. E. Clayton et al., Phys. Rev. Lett. 88, 154801 (2002).

[9] M. J. Hogan et al., Phys. Rev. Lett. 90, 205002 (2003).

[10] B. E. Blue et al., Phys. Rev. Lett. 90, 214801 (2003).

[11] D. K. Johnson et al., Phys. Rev. Lett. 97, 175003 (2006).

[12] P. Muggli et al., Phys. Rev. Lett. 93, 014802 (2004).

[13] M. J. Hogan et al., Phys. Rev. Lett. 95, 054802 (2005).

[14] I. Blumenfeld et al., Nature (London) 445, 741 (2007).

[15] A. V. Burdakov et al., Plasma Phys. Rep. 31, 292 (2005).

[16] A. V. Petrenko et al., in Proceedings of the 9th European Particle Accelerator Conference (Lucerne, 2004), p. 740.

[17] K. V. Lotov, Phys. Rev. ST Accel. Beams 6, 061301 (2003).

[18] K. V. Lotov, Phys. Plasmas 5, 785 (1998).

[19] K. V. Lotov, Phys. Rev. E 69, 046405 (2004).

[20] K. V. Lotov, Phys. Plasmas 12, 053105 (2005).

[21] J. B. Rosenzweig, B. Breizman, T. Katsouleas, and J. J. Su, Phys. Rev. A 44, R6189 (1991). 